\title{
Treatment of Refractory Chylous Ascites with an Innovative Peritoneovenous Shunt: Temporary Usage of a Continuous Renal Replacement System: A Case Report
}

\author{
Jiyoun Park, M.D., Jae Jun Lee, M.D., Jung Hee Lee, M.D., Young Mog Shim, M.D., Ph.D. \\ Department of Thoracic and Cardiovascular Surgery, Samsung Medical Center, Sungkyunkwan University School of Medicine, Seoul, Korea
}

ARTICLE INFO

Received August 9, 2021

Revised September 11, 2021

Accepted October 8, 2021

Corresponding author

Young Mog Shim

Tel 82-2-3410-3482

Fax 82-2-3410-1064

E-mail youngmog.shim@samsung.net ORCID

https://orcid.org/0000-0001-5924-9765

\begin{abstract}
Esophagectomy and esophageal reconstruction are commonly chosen as surgical options for esophageal cancer. However, prolonged untreated chyle leakage is associated with a poor prognosis. We report the case of a patient with refractory chylous ascites. To limit the ongoing fluid loss, we utilized the chylous ascites as an additional fluid source in a renal replacement therapy system. A continuous renal replacement therapy (CRRT) drainage system was modified to drain both the chylous ascites and venous blood. The ascites drainage rate was determined empirically and regulated by a dial-flow extension set. The CRRT mode was set to continuous venovenous hemodiafiltration and maintained for 7 days. After the patient was weaned from CRRT, ascites did not reaccumulate, and the patient's general condition improved dramatically. No infections related to the system occurred. This procedure temporarily alleviates symptoms and provides more time for alternative treatment strategies.
\end{abstract}

Keywords: Chylous ascites, Chylothorax, Peritoneovenous shunt, Esophageal neoplasms, Continuous hemodiafiltration shunt, Case report

\section{Case report}

A 67-year-old man with liver cirrhosis presented with dysphagia for solid foods. Esophagogastroduodenoscopy showed an ulcerative fungating mass $25-30 \mathrm{~cm}$ from the incisor, which was confirmed as squamous cell carcinoma on biopsy. Computed tomography and integrated positron emission tomography/computed tomography showed a locoregional tumor with paraesophageal lymph node metastasis, diagnosed as clinical T3N1M0 according to the American Joint Committee on Cancer, eighth edition. Before surgical esophagectomy, the patient completed neoadjuvant concurrent chemoradiotherapy in accordance with the recommendations of the 2018 National Comprehensive Cancer Network guideline for esophageal cancer. After 1 month, the patient underwent complete surgical resection (the Ivor Lewis operation).

Transthoracic esophagectomy, esophagogastrostomy, and 2-field (abdominal and mediastinal) lymph node dissection were performed. During dissection of the mediastinal lymph node, a lymphatic duct was injured, and the thoracic lymphatic ducts were ligated to prevent chylothorax. A pathologic examination confirmed ypT2N1M0 squamous cell carcinoma of the middle thoracic section of the esophagus.

The immediate postoperative course was uncomplicated. The anastomosis site was confirmed clear on an endoscopic assessment on postoperative day 3 , and the patient started eating on postoperative day 4. Bilateral pleural effusions then developed with mild abdominal distension. We inserted chest tubes bilaterally. Analysis of the pleural fluid revealed an elevated triglyceride level $(151 \mathrm{mg} / \mathrm{dL})$. The total amount of pleural drainage was $300-400 \mathrm{~mL} /$ day. The patient tried a fat-free diet after fasting for 7 days. The chylothorax improved, and the patient was discharged 40 days after surgery with instructions to follow a low-fat diet.

On the fifth day after discharge, the patient was readmitted to the emergency room with dyspnea and a large amount of right pleural effusion (Fig. 1). Despite draining the pleural effusion again, respiratory failure progressed, 


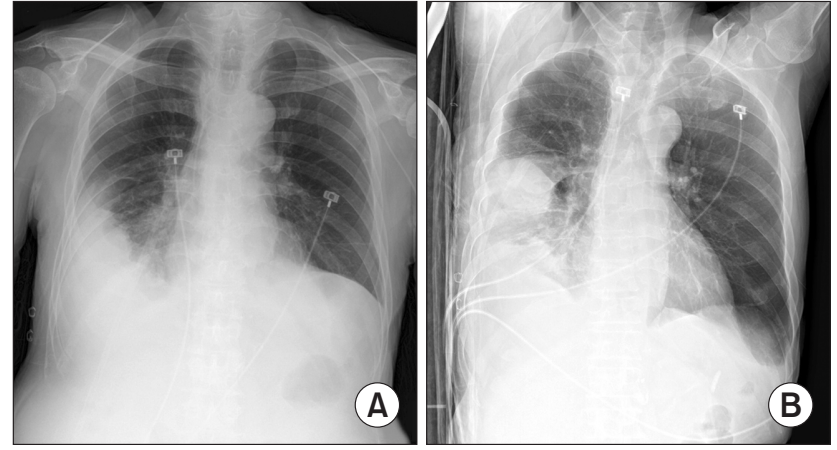

Fig. 1. Chest radiograph of the patient showing a right pleural effusion, which was drained (approximately 1,000 mL) after chest tubes were inserted. (A) Posteroanterior view. (B) Right lateral decubitus view.

and the patient was intubated. After 7 days of ventilatory support, respiration improved. The patient was weaned from the ventilator and extubated. However, progressive chylous ascites with severe abdominal distension developed, and respiratory failure recurred. The patient was intubated again, and a tracheostomy was created. Lymphangiography revealed a leak in the lymphatic duct adjacent to the common hepatic artery, and selective lymphatic duct embolization of the lesion was successfully performed. However, the patient's abdominal distension worsened. Surgical exploration of the abdomen was performed, and multiple leaks near the cisterna chyli were identified. The gross points of leakage were ligated, and hemostatic patches were applied elsewhere. However, the chylous ascites did not improve as expected after surgery. Because the patient could not be removed from the ventilator due to the chylous ascites, repeated paracentesis was required to relieve the severe dyspnea. Drainage of at least $2 \mathrm{~L}$ of ascites fluid was required to relieve his symptoms for 24 hours; we carefully drained 2-3 L of ascites every other day. Repeated paracentesis caused hypovolemia and hypotension despite the compensated intravascular volume replacement. Generalized edema and prerenal acute kidney injury developed, necessitating continuous renal replacement therapy (CRRT).

Because the ongoing loss of lymph into the peritoneal cavity complicated fluid therapy during CRRT, we experimentally utilized the chylous ascites fluid as an additional fluid source for renal replacement therapy. A 7F dual lumen catheter was inserted at the counter-McBurney point for ascites drainage. The CRRT drainage system was modified to drain both the chylous ascites and venous blood through a Hickman catheter in the jugular vein (Fig. 2). A dial-flow extension set was connected to the abdominal $7 \mathrm{~F}$

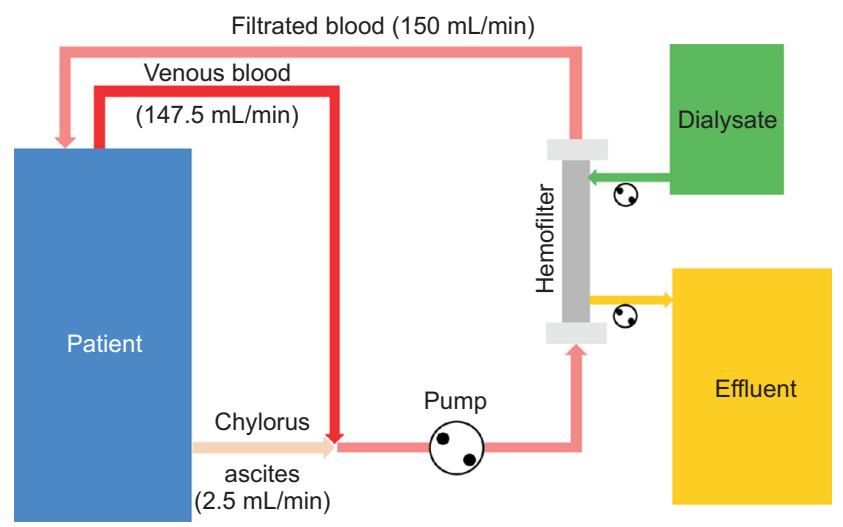

Fig. 2. Diagram of the continuous hemodiafiltration shunt used during continuous renal replacement therapy. If the total rate of infusion into the intravascular system from outside the patient was $100 \mathrm{~mL} / \mathrm{hr}$, the fluid removal rate would be set at $100 \mathrm{~mL} / \mathrm{hr}$. Chylous ascites in the peritoneum is like a reservoir for the extravascular system. Therefore, we used this extra volume for fluid replacement therapy until this reservoir was depleted. When hypotension necessitated rapid volume infusion, the speed of chylous ascites drainage was increased temporarily by dial-flow extension to over $300 \mathrm{~mL} / \mathrm{hr}$.

catheter to regulate the rate of ascites drainage. The basic total blood flow through the CRRT system was $150 \mathrm{~mL} /$ min. Assuming that the quantity of chylous ascites was $2-3$ $\mathrm{L}$ per day, we set the basic ascites drainage rate at $150 \mathrm{~mL} /$ $\mathrm{hr}$. If the chylous fluid leaked into the peritoneum at 100 $\mathrm{mL} / \mathrm{hr}$, a total of $50 \mathrm{~mL}$ of chylous ascites per hour would enter the intravascular volume system. The CRRT mode was set as continuous venovenous hemodiafiltration. Except for the chylous ascites, all other fluids entering the intravascular volume system from outside were included in the fluid removal rate in the CRRT (so-called "net zero"). We maintained this system for 7 days, at which point the acute kidney injury improved.

After the patient was weaned from CRRT, we left the abdominal drain catheter in place for 10 days in preparation to restart CRRT. Abdominal distension and ascites did not recur. Furthermore, the patient's general condition improved dramatically. No infections or embolic events related to the system occurred. The patient underwent surgical jejunostomy after recurrent aspiration during weaning from the ventilator. Eventually, the patient was successfully weaned from the ventilator and discharged 196 days after readmission with a normal diet through a jejunostomy tube.

This study was approved by the Institutional Review Board of Samsung Medical Center (IRB approval no., 2020-10-009). 


\section{Discussion}

In localized esophageal cancer, esophagectomy and gastrointestinal tract reconstruction are common surgical treatment options. During surgery, lymph node dissection is commonly performed for precise staging and prognosis, and the thoracic lymphatic ducts can be damaged. Thus, postoperative chyle leakage from those damaged ducts is not rare, but if the leakage is not treated appropriately, related complications such as hypovolemia, electrolyte imbalance, malnutrition, high infection risk, and sepsis can lead to mortality in $50 \%$ of cases [1]. Chylous ascites is less common than chylothorax because of the presence of positive pressure. The degree of chyle leakage depends on the underlying disease, such as liver cirrhosis, progression of esophageal cancer, neoadjuvant chemoradiation, and surgical extent. In addition to lymphatic duct injury, lymphoperitoneal fistula can cause chyle leakage. The principal management goals are to maintain nutrition and reduce the leakage of chyle. The first treatment option is a dietary approach. When chyle leakage is massive ( $>1 \mathrm{~L} /$ day), strict fasting and total parenteral nutrition are basic options for treatment that are noninvasive and reliable [2]. When chyle leakage is minor $(<500 \mathrm{~mL} /$ day), middle-chain triglyceride meals and low-fat or fat-free meals are feeding options. The second treatment option is the pharmacologic approach, which uses octreotide to treat chyle leakage.

The third option is surgical intervention, such as lymphatic duct embolization. Surgical ligation of lymphatic ducts is challenging. The points of lymphatic leakage are difficult to locate visually. Furthermore, complete ligation of the lymphatic ducts is uncertain because the ducts have complex connections [3]. Furthermore, the cisterna chyli, which is the main pathway of the lymphatic duct in the peritoneum, is not easily visualized. If chyle leakage continues, the use of a peritoneovenous shunt (PVS) is a palliative option [4]. The Denver shunt, introduced in the 1990s, is a PVS that is used mostly for the management of refractory nonchylous ascites. Relieving symptoms caused by abdominal overdistension can improve the patient's quality of life and aid in ventilator weaning. However, the Denver shunt is not available in Korea. Because all attempts to stop chyle leakage in our patient failed, we considered another PVS-like approach, a peritoneosaphenous vein shunt. However, the burden related to surgery would have been excessive for this patient. Coincidentally, the patient needed CRRT for acute kidney injury and volume management. We therefore used the CRRT machine as a large extracorporeal PVS. Since our goal was to remove ap- proximately $3 \mathrm{~L}$ of ascites per day, the flow rate was calculated to be about $2.5 \mathrm{~mL} / \mathrm{min}$, so we thought that a $7 \mathrm{~F}$ catheter would be sufficient. Another reason for selecting a smaller catheter was that the patient had severe abdominal distension and persistent ascites, and a wider catheter insertion site would increase the risk of catheter-related infection.

The use of a continuous hemodiafiltration shunt (CHDFS) with a CRRT machine has several advantages. First, a CHDFS is easily applied in any patient who needs shortterm palliative drainage, and it is less invasive than PVS application. However, the long-term use of a CHDFS is inappropriate because of the increased economic burden and the inability to use the shunt at home. Second, nutrients (including protein) lost by leakage into the peritoneum can be returned to the systemic circulation. It is well known that CRRT causes loss of amino acids, vitamins, and micro- and macro-elements. Although studies have shown that nutrient loss increases as the CRRT dose increases, the differences between CRRT types or techniques for preventing nutrient loss have not yet been clearly elucidated [5-8]. Numerous studies have suggested that nutrients should be supplied through either enteral or parenteral routes as a countermeasure against nutrient loss $[5,6]$. In this case, the patient had abdominal distension and severe dyspnea due to chylous ascites and required 2-3 L of paracentesis every other day. When this system was applied to CRRT, large proteins (e.g., albumin) were hardly filtered, so they could be returned to the patient. This was also the case for other middle- and low-molecular-weight proteins such as globulin, despite some losses by convection; rather than throwing away all the paracentesis fluid, we thought it would be helpful to put it back into the patient. Third, whereas the Denver shunt is contraindicated in patients with heart failure because intravascular volume overload can occur, the CHDFS can control volume status by ultrafiltration, making it useful in patients with heart failure. Fourth, a frequent adverse event with the Denver shunt is shunt occlusion [9]. In contrast, if the CHDFS becomes occluded, the circuit can be changed.

The CHDFS does have some limitations. First, because the abdominal drain catheter is inserted directly into the abdomen, the drainage catheter can be a route of peritoneal or blood infection. In this case, while maintaining the CRRT system, there were no suspicious findings of catheter-related infection, but Stenotrophomonas maltophilia was identified in an endotracheal culture before application of this system and antibiotics were used for pneumonia. The use of these antibiotics may have prevented cathe- 
ter-related infections. Second, unlike the Denver shunt, the CHDFS can be used only temporarily. Furthermore, frequent changes of the circuit are not cost-effective.

In conclusion, the use of a PVS and a CHDFS with a CRRT machine is not a long-term therapeutic option, but can be a valuable option for conservative treatment of refractory chylous ascites. This innovative design can relieve patients' symptoms and provide time for injured lymphatic ducts to recover.

\section{Conflict of interest}

No potential conflict of interest relevant to this article was reported.

\section{ORCID}

Jiyoun Park: https://orcid.org/0000-0002-6668-1406

Jae Jun Lee: https://orcid.org/0000-0003-0466-7630

Jung Hee Lee: https://orcid.org/0000-0002-5070-8248

Young Mog Shim: https://orcid.org/0000-0001-5924-9765

\section{References}

1. Lagarde SM, Omloo JM, de Jong K, Busch OR, Obertop H, van
Lanschot JJ. Incidence and management of chyle leakage after esophagectomy. Ann Thorac Surg 2005;80:449-54.

2. Weijs TJ, Ruurda JP, Broekhuizen ME, Bracco Gartner TC, van Hillegersberg R. Outcome of a step-up treatment strategy for chyle leakage after esophagectomy. Ann Thorac Surg 2017;104:477-84.

3. Yang YH, Park SY, Kim DJ. Chyle leakage after esophageal cancer surgery. Korean J Thorac Cardiovasc Surg 2020;53:191-9.

4. Yarmohammadi H, Brody LA, Erinjeri JP, et al. Therapeutic application of percutaneous peritoneovenous (Denver) shunt in treating chylous ascites in cancer patients. J Vasc Interv Radiol 2016;27:665-73.

5. Onichimowski D, Goraj R, Jalali R, Grabala J, Mayzner-Zawadzka E, Czuczwar M. Practical issues of nutrition during continuous renal replacement therapy. Anaesthesiol Intensive Ther 2017;49:309-16.

6. Honore PM, De Waele E, Jacobs R, et al. Nutritional and metabolic alterations during continuous renal replacement therapy. Blood Purif 2013;35:279-84.

7. VA/NIH Acute Renal Failure Trial Network, Palevsky PM, Zhang $\mathrm{JH}$, et al. Intensity of renal support in critically ill patients with acute kidney injury. N Engl J Med 2008;359:7-20.

8. RENAL Replacement Therapy Study Investigators, Bellomo R, Cass $\mathrm{A}$, et al. Intensity of continuous renal-replacement therapy in critically ill patients. N Engl J Med 2009;361:1627-38.

9. Huang Y, Gloviczki P, Duncan AA, et al. Management of refractory chylous ascites with peritoneovenous shunts. J Vasc Surg Venous Lymphat Disord 2017;5:538-46. 